Acta Crystallographica Section E

Structure Reports

Online

ISSN 1600-5368

\section{2-((Z)-\{3-[(Z)-(2-Hydroxy-5-methyl- benzylidene)amino]-2,2-dimethyl- propyl\}iminomethyl)-4-methylphenol}

\section{Reza Kia, ${ }^{\text {a,b* }}$ Hadi Kargar, ${ }^{c}$ Valiollah Mirkhani, ${ }^{d}$ Fatemeh Ganjic and Muhammad Nawaz Tahir ${ }^{\mathrm{e} *}$}

a Department of Chemistry, Science and Research Branch, Islamic Azad University, Tehran, Iran, ${ }^{\mathbf{b}} \mathrm{X}$-ray Crystallography Laboratory, Plasma Physics Research Center, Science and Research Branch, Islamic Azad University, Tehran, Iran, ${ }^{\mathbf{c}}$ Department of Chemistry, School of Science, Payame Noor University (PNU), Ardakan, Yazd, Iran,

dDepartment of Chemistry, University of Isfahan, Isfahan, 81746-73441, Iran, and $\mathbf{e}^{\mathbf{e}}$ Department of Physics, University of Sargodha, Punjab, Pakistan

Correspondence e-mail: rkia@srbiau.ac.ir, zsrkk@yahoo.com,

dmntahir_uos@yahoo.com

Received 4 December 2010; accepted 9 December 2010

Key indicators: single-crystal X-ray study; $T=296 \mathrm{~K}$; mean $\sigma(\mathrm{C}-\mathrm{C})=0.005 \AA$;

$R$ factor $=0.044 ; w R$ factor $=0.112 ;$ data-to-parameter ratio $=9.5$.

In the title compound, $\mathrm{C}_{21} \mathrm{H}_{26} \mathrm{~N}_{2} \mathrm{O}_{2}$, the dihedral angle between the two benzene rings is $73.47(16)^{\circ}$. Strong intramolecular $\mathrm{O}-\mathrm{H} \cdots \mathrm{N}$ hydrogen bonds generate $S(6)$ ring motifs. The substituted benzene rings are twisted around the central quaternary $\mathrm{C}$ atom in opposite directions, making a vault geometry.

\section{Related literature}

For standard bond lengths, see: Allen et al. (1987). For hydrogen-bond motifs, see: Bernstein et al. (1995). For related structures, see: Kargar et al. (2009, 2010).

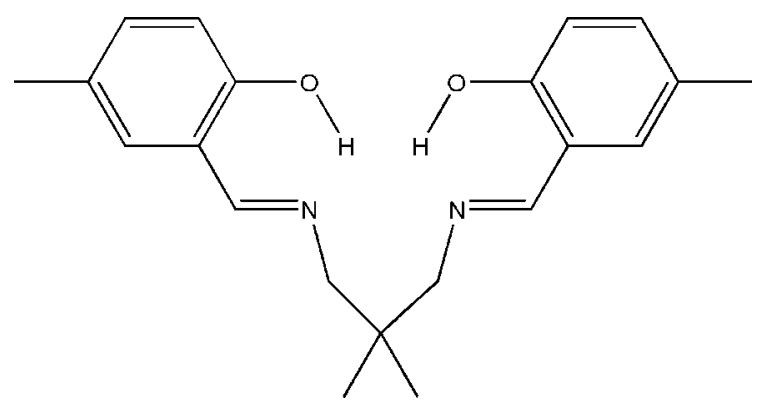

\section{Experimental}

Crystal data

$\mathrm{C}_{21} \mathrm{H}_{26} \mathrm{~N}_{2} \mathrm{O}_{2}$

Orthorhombic, $P 2_{1} 2_{1} 2_{1}$

$a=5.8950(3) \AA$

$b=17.8634(10) \AA$

$c=18.2140$ (11) $\AA$

$V=1918.02(19) \AA^{3}$

$Z=4$

Mo $K \alpha$ radiation

$\mu=0.08 \mathrm{~mm}^{-1}$

$T=296 \mathrm{~K}$

$0.30 \times 0.18 \times 0.12 \mathrm{~mm}$

Data collection

Bruker SMART APEXII CCD

area-detector diffractometer

Absorption correction: multi-scan

(SADABS; Bruker, 2005)

$T_{\min }=0.978, T_{\max }=0.991$

16208 measured reflections 2199 independent reflections 1368 reflections with $I>2 \sigma(I)$

$R_{\text {int }}=0.062$

Refinement

$R\left[F^{2}>2 \sigma\left(F^{2}\right)\right]=0.044$

$w R\left(F^{2}\right)=0.112$

$S=1.03$

2199 reflections

231 parameters

$\mathrm{H}$-atom parameters constrained

$\Delta \rho_{\max }=0.15{\mathrm{e} \AA^{-3}}^{-3}$

$\Delta \rho_{\min }=-0.12 \mathrm{e}^{-3}$

Table 1

Hydrogen-bond geometry $\left(\AA{ }^{\circ}\right)$.

\begin{tabular}{lllll}
\hline$D-\mathrm{H} \cdots A$ & $D-\mathrm{H}$ & $\mathrm{H} \cdots A$ & $D \cdots A$ & $D-\mathrm{H} \cdots A$ \\
\hline $\mathrm{O} 1-\mathrm{H} 1 A \cdots \mathrm{N} 1$ & 0.82 & 1.89 & $2.620(3)$ & 147 \\
$\mathrm{O} 2-\mathrm{H} 2 A \cdots \mathrm{N} 2$ & 0.82 & 1.88 & $2.609(4)$ & 147 \\
\hline
\end{tabular}

Data collection: APEX2 (Bruker, 2005); cell refinement: SAINT (Bruker, 2005); data reduction: $S A I N T$; $\operatorname{program}(\mathrm{s})$ used to solve structure: SHELXTL (Sheldrick, 2008); program(s) used to refine structure: SHELXTL; molecular graphics: SHELXTL; software used to prepare material for publication: SHELXTL and PLATON (Spek, 2009).

RK thanks the Science and Research Branch, Islamic Azad University. $\mathrm{HK}$ and FG thank PNU for financial support. MNT thanks the University of Sargodha, Pakistan, for the research facilities.

Supplementary data and figures for this paper are available from the IUCr electronic archives (Reference: GW2096).

\title{
References
}

Allen, F. H., Kennard, O., Watson, D. G., Brammer, L., Orpen, A. G. \& Taylor, R. (1987). J. Chem. Soc. Perkin Trans. 2, pp. S1-19.

Bernstein, J., Davis, R. E., Shimoni, L. \& Chang, N.-L. (1995). Angew. Chem. Int. Ed. Engl. 34, 1555-1573.

Bruker (2005). APEX2, SAINT and SADABS. Bruker AXS Inc., Madison, Wisconsin, USA.

Kargar, H., Kia, R., Jamshidvand, A. \& Fun, H.-K. (2009). Acta Cryst. E65, o776-o777.

Kargar, H., Kia, R., Ullah Khan, I. \& Sahraei, A. (2010). Acta Cryst. E66, o539.

Sheldrick, G. M. (2008). Acta Cryst. A64, 112-122.

Spek, A. L. (2009). Acta Cryst. D65, 148-155. 


\title{
supporting information
}

Acta Cryst. (2011). E67, o130 [https://doi.org/10.1107/S1600536810051688]

\section{2-((Z)-\{3-[(Z)-(2-Hydroxy-5-methylbenzylidene)amino]-2,2-dimethylpropyl\}- iminomethyl)-4-methylphenol}

\author{
Reza Kia, Hadi Kargar, Valiollah Mirkhani, Fatemeh Ganji and Muhammad Nawaz Tahir
}

\section{S1. Comment}

Schiff base ligands are one of the most prevalent systems in coordination chemistry. As part of a general study of tetradenate Schiff bases (Kargar et al. 2009; Kargar et al. 2010), we have determined the crystal structure of the title compound.

The asymmetric unit of the title compound, Fig. 1, comprises a potentially tetradenate Schiff base ligand. The bond lengths (Allen et al., 1987) and angles are within the normal ranges. The dihedral angle between the two phenyl rings is $73.47(16)^{\circ}$. Strong intramolecular $\mathrm{O}-\mathrm{H} \cdots \mathrm{N}$ hydrogen bonds generate $S(6)$ ring motifs (Bernstein et al., 1995). The title compound has a skew geometry. In the absence of sufficient anomalous scattering the absolute structure could not be determined.

\section{S2. Experimental}

The title compound was synthesized by adding 5 -methyl-salicylaldehyde $(4 \mathrm{mmol})$ to a solution of 2,2'-dimethylpropylenediamine $(2 \mathrm{mmol})$ in ethanol $(20 \mathrm{ml})$. The mixture was refluxed with stirring for half an hour. The resultant yellow solution was filtered. Yellow single crystals of the title compound suitable for $X$-ray structure determination were recrystallized from ethanol by slow evaporation of the solvents at room temperature over several days.

\section{S3. Refinement}

$\mathrm{H}$ atoms of the hydroxy groups were located by a rotating $\mathrm{O}-\mathrm{H}$ group and constraied to refine with the parent atoms with $\mathrm{U}_{\text {iso }}(\mathrm{H})=1.5 \mathrm{U}_{\text {eq }}(\mathrm{O})$, see Table 1 . The remaining $\mathrm{H}$ atoms were positioned geometrically with $\mathrm{C}-\mathrm{H}=0.93-0.97 \AA$ and included in a riding model approximation with $\mathrm{U}_{\text {iso }}(\mathrm{H})=1.2$ or $1.5 \mathrm{U}_{\mathrm{eq}}(\mathrm{C})$. A rotating group model was used for the methyl groups of the benzene rings. In the absence of sufficient anomalous scattering the absolute structure could not be determined and 1580 Friedel pairs were merged. 


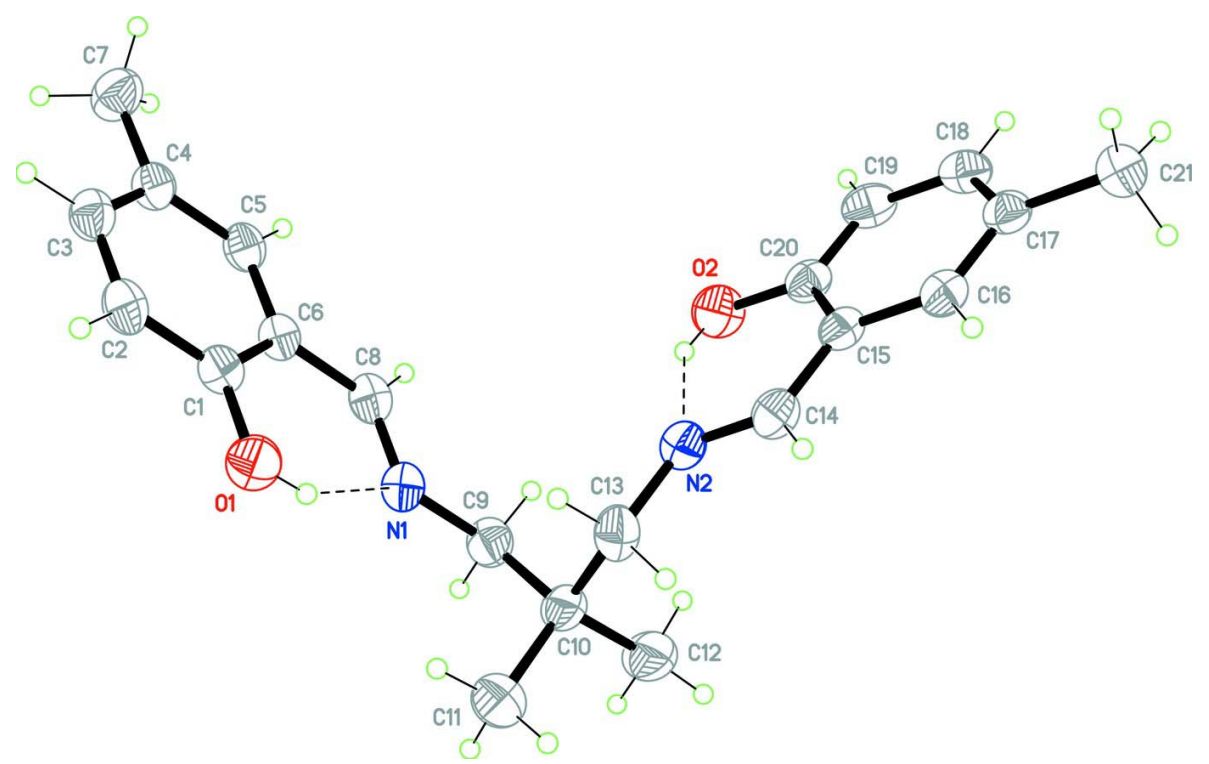

Figure 1

The asymmetric unit of the title compound, showing $30 \%$ probability displacement ellipsoids and the atomic numbering. Intramolecular hydrogen bonds are drawn as dashed lines.

2-((Z)-\{3-[(Z)-(2-Hydroxy-5-methylbenzylidene)amino]- 2,2-dimethylpropyl\}iminomethyl)-4-methylphenol

\section{Crystal data}

$\mathrm{C}_{21} \mathrm{H}_{26} \mathrm{~N}_{2} \mathrm{O}_{2}$

$M_{r}=338.44$

Orthorhombic, $P 2{ }_{1} 2_{1} 2_{1}$

Hall symbol: P $2 \mathrm{ac} 2 \mathrm{ab}$

$a=5.8950(3) \AA$

$b=17.8634(10) \AA$

$c=18.2140(11) \AA$

$V=1918.02(19) \AA^{3}$

$Z=4$

\section{Data collection}

\section{Bruker SMART APEXII CCD area-detector} diffractometer

Radiation source: fine-focus sealed tube

Graphite monochromator

$\varphi$ and $\omega$ scans

Absorption correction: multi-scan

(SADABS; Bruker, 2005)

$T_{\min }=0.978, T_{\max }=0.991$

\section{Refinement}

\section{Refinement on $F^{2}$}

Least-squares matrix: full

$R\left[F^{2}>2 \sigma\left(F^{2}\right)\right]=0.044$

$w R\left(F^{2}\right)=0.112$

$S=1.03$

2199 reflections

231 parameters
$F(000)=728$

$D_{\mathrm{x}}=1.172 \mathrm{Mg} \mathrm{m}^{-3}$

Mo $K \alpha$ radiation, $\lambda=0.71073 \AA$

Cell parameters from 2150 reflections

$\theta=2.5-29.8^{\circ}$

$\mu=0.08 \mathrm{~mm}^{-1}$

$T=296 \mathrm{~K}$

Plate, yellow

$0.30 \times 0.18 \times 0.12 \mathrm{~mm}$

16208 measured reflections

2199 independent reflections

1368 reflections with $I>2 \sigma(I)$

$R_{\text {int }}=0.062$

$\theta_{\max }=26.0^{\circ}, \theta_{\min }=2.2^{\circ}$

$h=-7 \rightarrow 7$

$k=-22 \rightarrow 22$

$l=-22 \rightarrow 22$

0 restraints

Primary atom site location: structure-invariant direct methods

Secondary atom site location: difference Fourier map

Hydrogen site location: inferred from neighbouring sites 
H-atom parameters constrained

$w=1 /\left[\sigma^{2}\left(F_{\mathrm{o}}^{2}\right)+(0.0485 P)^{2}+0.082 P\right]$

where $P=\left(F_{\mathrm{o}}^{2}+2 F_{\mathrm{c}}^{2}\right) / 3$

$(\Delta / \sigma)_{\max }<0.001$

$\Delta \rho_{\max }=0.15$ e $\AA^{-3}$
$\Delta \rho_{\min }=-0.12$ e $\AA^{-3}$

Extinction correction: SHELXTL (Sheldrick, 2008), $\mathrm{Fc}^{*}=\mathrm{kFc}\left[1+0.001 \mathrm{xFc}^{2} \lambda^{3} / \sin (2 \theta)\right]^{-1 / 4}$

Extinction coefficient: 0.013 (2)

Special details

Geometry. All esds (except the esd in the dihedral angle between two 1.s. planes) are estimated using the full covariance matrix. The cell esds are taken into account individually in the estimation of esds in distances, angles and torsion angles; correlations between esds in cell parameters are only used when they are defined by crystal symmetry. An approximate (isotropic) treatment of cell esds is used for estimating esds involving l.s. planes.

Refinement. Refinement of $\mathrm{F}^{2}$ against ALL reflections. The weighted R-factor wR and goodness of fit $\mathrm{S}$ are based on $\mathrm{F}^{2}$, conventional R-factors $R$ are based on $F$, with $F$ set to zero for negative $F^{2}$. The threshold expression of $\mathrm{F}^{2}>2 \operatorname{sigma}\left(\mathrm{F}^{2}\right)$ is used only for calculating R-factors(gt) etc. and is not relevant to the choice of reflections for refinement. R-factors based on $\mathrm{F}^{2}$ are statistically about twice as large as those based on F, and R- factors based on ALL data will be even larger.

Fractional atomic coordinates and isotropic or equivalent isotropic displacement parameters $\left(\AA^{2}\right)$

\begin{tabular}{|c|c|c|c|c|}
\hline & $x$ & $y$ & $z$ & $U_{\text {iso }} * / U_{\text {eq }}$ \\
\hline $\mathrm{O} 1$ & $0.4633(4)$ & $0.71477(13)$ & $0.18637(14)$ & $0.0691(7)$ \\
\hline H1A & 0.3887 & 0.6786 & 0.1999 & $0.104 *$ \\
\hline $\mathrm{O} 2$ & -0.1798 & $0.44857(14)$ & $0.02145(14)$ & $0.0769(7)$ \\
\hline $\mathrm{H} 2 \mathrm{~A}$ & -0.0936 & 0.4643 & 0.0533 & $0.115^{*}$ \\
\hline N1 & $0.1130(4)$ & $0.62471(14)$ & $0.19160(15)$ & $0.0576(7)$ \\
\hline $\mathrm{N} 2$ & $0.1758(5)$ & $0.44863(14)$ & $0.10671(15)$ & $0.0589(7)$ \\
\hline $\mathrm{C} 1$ & $0.3362(5)$ & $0.75772(18)$ & $0.14103(17)$ & $0.0513(8)$ \\
\hline $\mathrm{C} 2$ & 0.4215 & $0.82499(18)$ & 0.11644 (19) & $0.0593(9)$ \\
\hline $\mathrm{H} 2$ & 0.5650 & 0.8401 & 0.1316 & $0.071^{*}$ \\
\hline $\mathrm{C} 3$ & $0.2994(6)$ & 0.87012 (19) & $0.07010(18)$ & $0.0593(9)$ \\
\hline $\mathrm{H} 3$ & 0.3614 & 0.9153 & 0.0545 & $0.071 *$ \\
\hline $\mathrm{C} 4$ & $0.0828(5)$ & $0.84926(18)$ & $0.04596(18)$ & $0.0558(9)$ \\
\hline $\mathrm{C} 5$ & $-0.0004(6)$ & $0.78143(17)$ & $0.07017(17)$ & $0.0544(8)$ \\
\hline H5 & -0.1421 & 0.7659 & 0.0537 & $0.065^{*}$ \\
\hline C6 & $0.1183(5)$ & $0.73540(17)$ & $0.11802(17)$ & $0.0487(8)$ \\
\hline $\mathrm{C} 7$ & $-0.0504(7)$ & 0.90009 (19) & -0.0039 (2) & $0.0777(11)$ \\
\hline $\mathrm{H} 7 \mathrm{~A}$ & 0.0048 & 0.8952 & -0.0533 & $0.117^{*}$ \\
\hline $\mathrm{H} 7 \mathrm{~B}$ & -0.0332 & 0.9510 & 0.0120 & $0.117^{*}$ \\
\hline $\mathrm{H} 7 \mathrm{C}$ & -0.2079 & 0.8865 & -0.0023 & $0.117^{*}$ \\
\hline $\mathrm{C} 8$ & $0.0162(6)$ & $0.66623(17)$ & $0.14440(18)$ & $0.0562(9)$ \\
\hline H8 & -0.1246 & 0.6520 & 0.1261 & $0.067^{*}$ \\
\hline C9 & $-0.0035(6)$ & $0.55714(17)$ & 0.21706 (19) & $0.0641(10)$ \\
\hline H9A & -0.1067 & 0.5401 & 0.1791 & $0.077^{*}$ \\
\hline H9B & -0.0929 & 0.5693 & 0.2601 & $0.077 *$ \\
\hline $\mathrm{C} 10$ & $0.1605(6)$ & $0.49409(17)$ & $0.23599(18)$ & $0.0615(9)$ \\
\hline C11 & $0.3201(7)$ & $0.5184(2)$ & $0.2980(2)$ & $0.0892(13)$ \\
\hline H11A & 0.2336 & 0.5276 & 0.3417 & $0.134^{*}$ \\
\hline H11B & 0.3982 & 0.5633 & 0.2838 & $0.134^{*}$ \\
\hline $\mathrm{H} 11 \mathrm{C}$ & 0.4286 & 0.4794 & 0.3072 & $0.134^{*}$ \\
\hline $\mathrm{C} 12$ & $0.0218(8)$ & $0.4259(2)$ & $0.2593(2)$ & $0.0891(13)$ \\
\hline
\end{tabular}




$\begin{array}{lllll}\text { H12A } & 0.1216 & 0.3842 & 0.2673 & 0.134^{*} \\ \text { H12B } & -0.0850 & 0.4136 & 0.2214 & 0.134^{*} \\ \text { H12C } & -0.0582 & 0.4370 & 0.3039 & 0.134^{*} \\ \text { C13 } & 0.3085(6) & 0.47518(18) & 0.16952(19) & 0.0640(10) \\ \text { H13A } & 0.3929 & 0.5194 & 0.1551 & 0.077^{*} \\ \text { H13B } & 0.4171 & 0.4369 & 0.1833 & 0.077^{*} \\ \text { C14 } & 0.2562(6) & 0.39671(18) & 0.06619(18) & 0.0571(9) \\ \text { H14 } & 0.3962 & 0.3762 & 0.0784 & 0.069^{*} \\ \text { C15 } & 0.1397(5) & 0.36831(16) & 0.00220(18) & 0.0512(8) \\ \text { C16 } & 0.2388(6) & 0.31298(17) & -0.04090(17) & 0.0542(8) \\ \text { H16 } & 0.3790 & 0.2940 & -0.0267 & 0.065^{*} \\ \text { C17 } & 0.1400(6) & 0.28490(17) & -0.10341(18) & 0.0570(9) \\ \text { C18 } & -0.0714(6) & 0.3128(2) & -0.1220(2) & 0.0638(10) \\ \text { H18 } & -0.1438 & 0.2943 & -0.1636 & 0.077^{*} \\ \text { C19 } & -0.1782(6) & 0.3673(2) & -0.08042(19) & 0.0648(9) \\ \text { H19 } & -0.3205 & 0.3850 & -0.0941 & 0.078^{*} \\ \text { C20 } & -0.0727(5) & 0.39531(18) & -0.01880(19) & 0.0542(9) \\ \text { C21 } & 0.2588(7) & 0.22644(19) & -0.14975(19) & 0.0775(12) \\ \text { H21A } & 0.3779 & 0.2497 & -0.1775 & 0.116^{*} \\ \text { H21B } & 0.1517 & 0.2039 & -0.1827 & 0.116^{*} \\ \text { H21C } & 0.3222 & 0.1886 & -0.1184 & 0.116^{*}\end{array}$

Atomic displacement parameters $\left(\AA^{2}\right)$

\begin{tabular}{lllllll}
\hline & $U^{11}$ & $U^{22}$ & $U^{33}$ & $U^{12}$ & $U^{13}$ & $U^{23}$ \\
\hline O1 & $0.0584(15)$ & $0.0711(17)$ & $0.0778(17)$ & $0.0012(12)$ & $-0.0145(14)$ & $0.0031(14)$ \\
O2 & $0.0690(16)$ & $0.0818(17)$ & $0.0798(19)$ & $0.0272(15)$ & $0.0009(14)$ & $-0.0003(14)$ \\
N1 & $0.0590(17)$ & $0.0501(16)$ & $0.0636(18)$ & $0.0000(13)$ & $0.0014(15)$ & $-0.0032(14)$ \\
N2 & $0.0615(17)$ & $0.0523(17)$ & $0.0629(18)$ & $0.0032(14)$ & $-0.0008(16)$ & $0.0023(15)$ \\
C1 & $0.0439(18)$ & $0.0545(19)$ & $0.056(2)$ & $0.0055(16)$ & $-0.0042(16)$ & $-0.0062(16)$ \\
C2 & $0.0503(19)$ & $0.060(2)$ & $0.068(2)$ & $-0.0066(17)$ & $-0.0024(18)$ & $-0.0138(18)$ \\
C3 & $0.057(2)$ & $0.056(2)$ & $0.065(2)$ & $-0.0064(17)$ & $0.0018(18)$ & $-0.0047(18)$ \\
C4 & $0.056(2)$ & $0.054(2)$ & $0.058(2)$ & $0.0018(16)$ & $0.0007(17)$ & $-0.0054(16)$ \\
C5 & $0.0467(18)$ & $0.055(2)$ & $0.061(2)$ & $-0.0020(16)$ & $-0.0037(17)$ & $-0.0096(17)$ \\
C6 & $0.0423(17)$ & $0.0497(18)$ & $0.0540(19)$ & $-0.0008(14)$ & $0.0011(16)$ & $-0.0062(16)$ \\
C7 & $0.078(3)$ & $0.070(2)$ & $0.085(3)$ & $-0.0059(19)$ & $-0.015(2)$ & $0.014(2)$ \\
C8 & $0.0485(19)$ & $0.057(2)$ & $0.063(2)$ & $-0.0020(16)$ & $0.0052(17)$ & $-0.0098(17)$ \\
C9 & $0.067(2)$ & $0.061(2)$ & $0.064(2)$ & $-0.0107(19)$ & $0.0100(19)$ & $-0.0015(17)$ \\
C10 & $0.072(2)$ & $0.052(2)$ & $0.060(2)$ & $-0.0139(19)$ & $-0.0039(19)$ & $0.0081(17)$ \\
C11 & $0.109(3)$ & $0.080(3)$ & $0.079(3)$ & $-0.012(2)$ & $-0.027(3)$ & $-0.002(2)$ \\
C12 & $0.116(3)$ & $0.077(3)$ & $0.074(3)$ & $-0.032(3)$ & $-0.009(3)$ & $0.016(2)$ \\
C13 & $0.060(2)$ & $0.052(2)$ & $0.080(3)$ & $-0.0010(17)$ & $-0.006(2)$ & $-0.0061(18)$ \\
C14 & $0.0495(19)$ & $0.054(2)$ & $0.068(2)$ & $0.0020(16)$ & $-0.0012(18)$ & $0.0062(18)$ \\
C15 & $0.0506(19)$ & $0.0491(18)$ & $0.0538(19)$ & $0.0021(15)$ & $0.0049(17)$ & $0.0105(16)$ \\
C16 & $0.0496(19)$ & $0.0534(19)$ & $0.059(2)$ & $0.0028(15)$ & $0.0031(17)$ & $0.0083(17)$ \\
C17 & $0.063(2)$ & $0.055(2)$ & $0.053(2)$ & $-0.0015(17)$ & $0.0069(18)$ & $0.0096(17)$ \\
C18 & $0.064(2)$ & $0.072(2)$ & $0.055(2)$ & $-0.0100(19)$ & $-0.0029(19)$ & $0.0109(18)$ \\
C19 & $0.0495(19)$ & $0.079(3)$ & $0.066(2)$ & $0.0022(19)$ & $-0.002(2)$ & $0.018(2)$ \\
& & & & & &
\end{tabular}




\begin{tabular}{|c|c|c|c|c|c|c|}
\hline $\mathrm{C} 20$ & $0.051(2)$ & 0.0555 (19) & $0.057(2)$ & $0.0067(16)$ & $0.0082(17)$ & $0.0106(17)$ \\
\hline $\mathrm{C} 21$ & $0.099(3)$ & $0.068(2)$ & $0.065(2)$ & $0.011(2)$ & $0.006(2)$ & -0.0031 \\
\hline
\end{tabular}

Geometric parameters $\left(\AA,{ }^{\circ}\right)$

\begin{tabular}{|c|c|c|c|}
\hline $\mathrm{O} 1-\mathrm{C} 1$ & $1.354(3)$ & $\mathrm{C} 10-\mathrm{C} 12$ & $1.527(5)$ \\
\hline $\mathrm{O} 1-\mathrm{H} 1 \mathrm{~A}$ & 0.8200 & $\mathrm{C} 10-\mathrm{C} 13$ & $1.530(4)$ \\
\hline $\mathrm{O} 2-\mathrm{C} 20$ & $1.357(4)$ & $\mathrm{C} 10-\mathrm{C} 11$ & $1.533(5)$ \\
\hline $\mathrm{O} 2-\mathrm{H} 2 \mathrm{~A}$ & 0.8200 & $\mathrm{C} 11-\mathrm{H} 11 \mathrm{~A}$ & 0.9600 \\
\hline $\mathrm{N} 1-\mathrm{C} 8$ & $1.271(4)$ & $\mathrm{C} 11-\mathrm{H} 11 \mathrm{~B}$ & 0.9600 \\
\hline $\mathrm{N} 1-\mathrm{C} 9$ & $1.464(4)$ & $\mathrm{C} 11-\mathrm{H} 11 \mathrm{C}$ & 0.9600 \\
\hline $\mathrm{N} 2-\mathrm{C} 14$ & $1.276(4)$ & $\mathrm{C} 12-\mathrm{H} 12 \mathrm{~A}$ & 0.9600 \\
\hline $\mathrm{N} 2-\mathrm{C} 13$ & $1.465(4)$ & $\mathrm{C} 12-\mathrm{H} 12 \mathrm{~B}$ & 0.9600 \\
\hline $\mathrm{C} 1-\mathrm{C} 2$ & $1.377(4)$ & $\mathrm{C} 12-\mathrm{H} 12 \mathrm{C}$ & 0.9600 \\
\hline $\mathrm{C} 1-\mathrm{C} 6$ & $1.409(4)$ & $\mathrm{C} 13-\mathrm{H} 13 \mathrm{~A}$ & 0.9700 \\
\hline $\mathrm{C} 2-\mathrm{C} 3$ & $1.371(4)$ & $\mathrm{C} 13-\mathrm{H} 13 \mathrm{~B}$ & 0.9700 \\
\hline $\mathrm{C} 2-\mathrm{H} 2$ & 0.9300 & $\mathrm{C} 14-\mathrm{C} 15$ & $1.445(4)$ \\
\hline $\mathrm{C} 3-\mathrm{C} 4$ & $1.401(4)$ & $\mathrm{C} 14-\mathrm{H} 14$ & 0.9300 \\
\hline $\mathrm{C} 3-\mathrm{H} 3$ & 0.9300 & $\mathrm{C} 15-\mathrm{C} 16$ & $1.391(4)$ \\
\hline $\mathrm{C} 4-\mathrm{C} 5$ & $1.380(4)$ & $\mathrm{C} 15-\mathrm{C} 20$ & $1.395(4)$ \\
\hline $\mathrm{C} 4-\mathrm{C} 7$ & $1.506(4)$ & $\mathrm{C} 16-\mathrm{C} 17$ & $1.374(4)$ \\
\hline $\mathrm{C} 5-\mathrm{C} 6$ & $1.388(4)$ & $\mathrm{C} 16-\mathrm{H} 16$ & 0.9300 \\
\hline $\mathrm{C} 5-\mathrm{H} 5$ & 0.9300 & $\mathrm{C} 17-\mathrm{C} 18$ & $1.384(5)$ \\
\hline $\mathrm{C} 6-\mathrm{C} 8$ & $1.456(4)$ & $\mathrm{C} 17-\mathrm{C} 21$ & $1.515(4)$ \\
\hline $\mathrm{C} 7-\mathrm{H} 7 \mathrm{~A}$ & 0.9600 & $\mathrm{C} 18-\mathrm{C} 19$ & $1.386(5)$ \\
\hline $\mathrm{C} 7-\mathrm{H} 7 \mathrm{~B}$ & 0.9600 & C18-H18 & 0.9300 \\
\hline $\mathrm{C} 7-\mathrm{H} 7 \mathrm{C}$ & 0.9600 & $\mathrm{C} 19-\mathrm{C} 20$ & $1.377(4)$ \\
\hline $\mathrm{C} 8-\mathrm{H} 8$ & 0.9300 & C19-H19 & 0.9300 \\
\hline $\mathrm{C} 9-\mathrm{C} 10$ & $1.524(5)$ & $\mathrm{C} 21-\mathrm{H} 21 \mathrm{~A}$ & 0.9600 \\
\hline C9-H9A & 0.9700 & $\mathrm{C} 21-\mathrm{H} 21 \mathrm{~B}$ & 0.9600 \\
\hline C9-H9B & 0.9700 & $\mathrm{C} 21-\mathrm{H} 21 \mathrm{C}$ & 0.9600 \\
\hline $\mathrm{C} 1-\mathrm{O} 1-\mathrm{H} 1 \mathrm{~A}$ & 109.5 & $\mathrm{C} 10-\mathrm{C} 11-\mathrm{H} 11 \mathrm{~B}$ & 109.5 \\
\hline $\mathrm{C} 20-\mathrm{O} 2-\mathrm{H} 2 \mathrm{~A}$ & 109.5 & H11A-C11-H11B & 109.5 \\
\hline $\mathrm{C} 8-\mathrm{N} 1-\mathrm{C} 9$ & $119.0(3)$ & $\mathrm{C} 10-\mathrm{C} 11-\mathrm{H} 11 \mathrm{C}$ & 109.5 \\
\hline $\mathrm{C} 14-\mathrm{N} 2-\mathrm{C} 13$ & $119.3(3)$ & $\mathrm{H} 11 \mathrm{~A}-\mathrm{C} 11-\mathrm{H} 11 \mathrm{C}$ & 109.5 \\
\hline $\mathrm{O} 1-\mathrm{C} 1-\mathrm{C} 2$ & $119.4(3)$ & $\mathrm{H} 11 \mathrm{~B}-\mathrm{C} 11-\mathrm{H} 11 \mathrm{C}$ & 109.5 \\
\hline $\mathrm{O} 1-\mathrm{C} 1-\mathrm{C} 6$ & $121.7(3)$ & $\mathrm{C} 10-\mathrm{C} 12-\mathrm{H} 12 \mathrm{~A}$ & 109.5 \\
\hline $\mathrm{C} 2-\mathrm{C} 1-\mathrm{C} 6$ & $118.9(3)$ & $\mathrm{C} 10-\mathrm{C} 12-\mathrm{H} 12 \mathrm{~B}$ & 109.5 \\
\hline $\mathrm{C} 3-\mathrm{C} 2-\mathrm{C} 1$ & $121.4(3)$ & $\mathrm{H} 12 \mathrm{~A}-\mathrm{C} 12-\mathrm{H} 12 \mathrm{~B}$ & 109.5 \\
\hline $\mathrm{C} 3-\mathrm{C} 2-\mathrm{H} 2$ & 119.3 & $\mathrm{C} 10-\mathrm{C} 12-\mathrm{H} 12 \mathrm{C}$ & 109.5 \\
\hline $\mathrm{C} 1-\mathrm{C} 2-\mathrm{H} 2$ & 119.3 & $\mathrm{H} 12 \mathrm{~A}-\mathrm{C} 12-\mathrm{H} 12 \mathrm{C}$ & 109.5 \\
\hline $\mathrm{C} 2-\mathrm{C} 3-\mathrm{C} 4$ & $121.0(3)$ & $\mathrm{H} 12 \mathrm{~B}-\mathrm{C} 12-\mathrm{H} 12 \mathrm{C}$ & 109.5 \\
\hline $\mathrm{C} 2-\mathrm{C} 3-\mathrm{H} 3$ & 119.5 & $\mathrm{~N} 2-\mathrm{C} 13-\mathrm{C} 10$ & $112.6(3)$ \\
\hline $\mathrm{C} 4-\mathrm{C} 3-\mathrm{H} 3$ & 119.5 & $\mathrm{~N} 2-\mathrm{C} 13-\mathrm{H} 13 \mathrm{~A}$ & 109.1 \\
\hline $\mathrm{C} 5-\mathrm{C} 4-\mathrm{C} 3$ & $117.2(3)$ & $\mathrm{C} 10-\mathrm{C} 13-\mathrm{H} 13 \mathrm{~A}$ & 109.1 \\
\hline $\mathrm{C} 5-\mathrm{C} 4-\mathrm{C} 7$ & $122.5(3)$ & $\mathrm{N} 2-\mathrm{C} 13-\mathrm{H} 13 \mathrm{~B}$ & 109.1 \\
\hline $\mathrm{C} 3-\mathrm{C} 4-\mathrm{C} 7$ & $120.3(3)$ & $\mathrm{C} 10-\mathrm{C} 13-\mathrm{H} 13 \mathrm{~B}$ & 109.1 \\
\hline
\end{tabular}




\begin{tabular}{|c|c|c|c|}
\hline $\mathrm{C} 4-\mathrm{C} 5-\mathrm{C} 6$ & $122.8(3)$ & $\mathrm{H} 13 \mathrm{~A}-\mathrm{C} 13-\mathrm{H} 13 \mathrm{~B}$ & 107.8 \\
\hline $\mathrm{C} 4-\mathrm{C} 5-\mathrm{H} 5$ & 118.6 & $\mathrm{~N} 2-\mathrm{C} 14-\mathrm{C} 15$ & $123.0(3)$ \\
\hline $\mathrm{C} 6-\mathrm{C} 5-\mathrm{H} 5$ & 118.6 & $\mathrm{~N} 2-\mathrm{C} 14-\mathrm{H} 14$ & 118.5 \\
\hline $\mathrm{C} 5-\mathrm{C} 6-\mathrm{C} 1$ & $118.6(3)$ & $\mathrm{C} 15-\mathrm{C} 14-\mathrm{H} 14$ & 118.5 \\
\hline $\mathrm{C} 5-\mathrm{C} 6-\mathrm{C} 8$ & $120.1(3)$ & $\mathrm{C} 16-\mathrm{C} 15-\mathrm{C} 20$ & $117.9(3)$ \\
\hline $\mathrm{C} 1-\mathrm{C} 6-\mathrm{C} 8$ & $121.3(3)$ & $\mathrm{C} 16-\mathrm{C} 15-\mathrm{C} 14$ & $120.4(3)$ \\
\hline $\mathrm{C} 4-\mathrm{C} 7-\mathrm{H} 7 \mathrm{~A}$ & 109.5 & $\mathrm{C} 20-\mathrm{C} 15-\mathrm{C} 14$ & $121.7(3)$ \\
\hline $\mathrm{C} 4-\mathrm{C} 7-\mathrm{H} 7 \mathrm{~B}$ & 109.5 & $\mathrm{C} 17-\mathrm{C} 16-\mathrm{C} 15$ & $123.3(3)$ \\
\hline $\mathrm{H} 7 \mathrm{~A}-\mathrm{C} 7-\mathrm{H} 7 \mathrm{~B}$ & 109.5 & $\mathrm{C} 17-\mathrm{C} 16-\mathrm{H} 16$ & 118.3 \\
\hline $\mathrm{C} 4-\mathrm{C} 7-\mathrm{H} 7 \mathrm{C}$ & 109.5 & $\mathrm{C} 15-\mathrm{C} 16-\mathrm{H} 16$ & 118.3 \\
\hline $\mathrm{H} 7 \mathrm{~A}-\mathrm{C} 7-\mathrm{H} 7 \mathrm{C}$ & 109.5 & $\mathrm{C} 16-\mathrm{C} 17-\mathrm{C} 18$ & $117.0(3)$ \\
\hline $\mathrm{H} 7 \mathrm{~B}-\mathrm{C} 7-\mathrm{H} 7 \mathrm{C}$ & 109.5 & $\mathrm{C} 16-\mathrm{C} 17-\mathrm{C} 21$ & $121.2(3)$ \\
\hline $\mathrm{N} 1-\mathrm{C} 8-\mathrm{C} 6$ & $122.2(3)$ & $\mathrm{C} 18-\mathrm{C} 17-\mathrm{C} 21$ & $121.9(3)$ \\
\hline $\mathrm{N} 1-\mathrm{C} 8-\mathrm{H} 8$ & 118.9 & $\mathrm{C} 17-\mathrm{C} 18-\mathrm{C} 19$ & $121.9(4)$ \\
\hline $\mathrm{C} 6-\mathrm{C} 8-\mathrm{H} 8$ & 118.9 & $\mathrm{C} 17-\mathrm{C} 18-\mathrm{H} 18$ & 119.0 \\
\hline $\mathrm{N} 1-\mathrm{C} 9-\mathrm{C} 10$ & $112.6(3)$ & $\mathrm{C} 19-\mathrm{C} 18-\mathrm{H} 18$ & 119.0 \\
\hline $\mathrm{N} 1-\mathrm{C} 9-\mathrm{H} 9 \mathrm{~A}$ & 109.1 & $\mathrm{C} 20-\mathrm{C} 19-\mathrm{C} 18$ & $119.7(3)$ \\
\hline $\mathrm{C} 10-\mathrm{C} 9-\mathrm{H} 9 \mathrm{~A}$ & 109.1 & $\mathrm{C} 20-\mathrm{C} 19-\mathrm{H} 19$ & 120.2 \\
\hline $\mathrm{N} 1-\mathrm{C} 9-\mathrm{H} 9 \mathrm{~B}$ & 109.1 & $\mathrm{C} 18-\mathrm{C} 19-\mathrm{H} 19$ & 120.2 \\
\hline $\mathrm{C} 10-\mathrm{C} 9-\mathrm{H} 9 \mathrm{~B}$ & 109.1 & $\mathrm{O} 2-\mathrm{C} 20-\mathrm{C} 19$ & $119.0(3)$ \\
\hline $\mathrm{H} 9 \mathrm{~A}-\mathrm{C} 9-\mathrm{H} 9 \mathrm{~B}$ & 107.8 & $\mathrm{O} 2-\mathrm{C} 20-\mathrm{C} 15$ & $120.8(3)$ \\
\hline $\mathrm{C} 9-\mathrm{C} 10-\mathrm{C} 12$ & $108.2(3)$ & $\mathrm{C} 19-\mathrm{C} 20-\mathrm{C} 15$ & $120.2(3)$ \\
\hline $\mathrm{C} 9-\mathrm{C} 10-\mathrm{C} 13$ & $110.2(3)$ & $\mathrm{C} 17-\mathrm{C} 21-\mathrm{H} 21 \mathrm{~A}$ & 109.5 \\
\hline $\mathrm{C} 12-\mathrm{C} 10-\mathrm{C} 13$ & $110.4(3)$ & $\mathrm{C} 17-\mathrm{C} 21-\mathrm{H} 21 \mathrm{~B}$ & 109.5 \\
\hline $\mathrm{C} 9-\mathrm{C} 10-\mathrm{C} 11$ & $110.3(3)$ & $\mathrm{H} 21 \mathrm{~A}-\mathrm{C} 21-\mathrm{H} 21 \mathrm{~B}$ & 109.5 \\
\hline $\mathrm{C} 12-\mathrm{C} 10-\mathrm{C} 11$ & $110.5(3)$ & $\mathrm{C} 17-\mathrm{C} 21-\mathrm{H} 21 \mathrm{C}$ & 109.5 \\
\hline $\mathrm{C} 13-\mathrm{C} 10-\mathrm{C} 11$ & $107.2(3)$ & $\mathrm{H} 21 \mathrm{~A}-\mathrm{C} 21-\mathrm{H} 21 \mathrm{C}$ & 109.5 \\
\hline $\mathrm{C} 10-\mathrm{C} 11-\mathrm{H} 11 \mathrm{~A}$ & 109.5 & $\mathrm{H} 21 \mathrm{~B}-\mathrm{C} 21-\mathrm{H} 21 \mathrm{C}$ & 109.5 \\
\hline $\mathrm{O} 1-\mathrm{C} 1-\mathrm{C} 2-\mathrm{C} 3$ & $179.6(3)$ & $\mathrm{C} 14-\mathrm{N} 2-\mathrm{C} 13-\mathrm{C} 10$ & $-142.8(3)$ \\
\hline $\mathrm{C} 6-\mathrm{C} 1-\mathrm{C} 2-\mathrm{C} 3$ & $-0.3(5)$ & $\mathrm{C} 9-\mathrm{C} 10-\mathrm{C} 13-\mathrm{N} 2$ & $-61.8(4)$ \\
\hline $\mathrm{C} 1-\mathrm{C} 2-\mathrm{C} 3-\mathrm{C} 4$ & $-0.1(5)$ & $\mathrm{C} 12-\mathrm{C} 10-\mathrm{C} 13-\mathrm{N} 2$ & $57.8(4)$ \\
\hline $\mathrm{C} 2-\mathrm{C} 3-\mathrm{C} 4-\mathrm{C} 5$ & $-0.6(5)$ & $\mathrm{C} 11-\mathrm{C} 10-\mathrm{C} 13-\mathrm{N} 2$ & $178.1(3)$ \\
\hline $\mathrm{C} 2-\mathrm{C} 3-\mathrm{C} 4-\mathrm{C} 7$ & $179.0(3)$ & $\mathrm{C} 13-\mathrm{N} 2-\mathrm{C} 14-\mathrm{C} 15$ & $-178.5(3)$ \\
\hline $\mathrm{C} 3-\mathrm{C} 4-\mathrm{C} 5-\mathrm{C} 6$ & $1.7(5)$ & $\mathrm{N} 2-\mathrm{C} 14-\mathrm{C} 15-\mathrm{C} 16$ & $178.3(3)$ \\
\hline $\mathrm{C} 7-\mathrm{C} 4-\mathrm{C} 5-\mathrm{C} 6$ & $-177.9(3)$ & $\mathrm{N} 2-\mathrm{C} 14-\mathrm{C} 15-\mathrm{C} 20$ & $-1.2(5)$ \\
\hline $\mathrm{C} 4-\mathrm{C} 5-\mathrm{C} 6-\mathrm{C} 1$ & $-2.1(4)$ & $\mathrm{C} 20-\mathrm{C} 15-\mathrm{C} 16-\mathrm{C} 17$ & $1.3(4)$ \\
\hline $\mathrm{C} 4-\mathrm{C} 5-\mathrm{C} 6-\mathrm{C} 8$ & $176.9(3)$ & $\mathrm{C} 14-\mathrm{C} 15-\mathrm{C} 16-\mathrm{C} 17$ & $-178.3(3)$ \\
\hline $\mathrm{O} 1-\mathrm{C} 1-\mathrm{C} 6-\mathrm{C} 5$ & $-178.6(3)$ & $\mathrm{C} 15-\mathrm{C} 16-\mathrm{C} 17-\mathrm{C} 18$ & $-1.7(5)$ \\
\hline $\mathrm{C} 2-\mathrm{C} 1-\mathrm{C} 6-\mathrm{C} 5$ & $1.3(4)$ & $\mathrm{C} 15-\mathrm{C} 16-\mathrm{C} 17-\mathrm{C} 21$ & $177.9(3)$ \\
\hline $\mathrm{O} 1-\mathrm{C} 1-\mathrm{C} 6-\mathrm{C} 8$ & $2.4(4)$ & $\mathrm{C} 16-\mathrm{C} 17-\mathrm{C} 18-\mathrm{C} 19$ & $1.0(5)$ \\
\hline $\mathrm{C} 2-\mathrm{C} 1-\mathrm{C} 6-\mathrm{C} 8$ & $-177.7(3)$ & $\mathrm{C} 21-\mathrm{C} 17-\mathrm{C} 18-\mathrm{C} 19$ & $-178.7(3)$ \\
\hline $\mathrm{C} 9-\mathrm{N} 1-\mathrm{C} 8-\mathrm{C} 6$ & $178.2(3)$ & $\mathrm{C} 17-\mathrm{C} 18-\mathrm{C} 19-\mathrm{C} 20$ & $0.2(5)$ \\
\hline $\mathrm{C} 5-\mathrm{C} 6-\mathrm{C} 8-\mathrm{N} 1$ & $-175.7(3)$ & $\mathrm{C} 18-\mathrm{C} 19-\mathrm{C} 20-\mathrm{O} 2$ & $-179.8(3)$ \\
\hline $\mathrm{C} 1-\mathrm{C} 6-\mathrm{C} 8-\mathrm{N} 1$ & $3.3(5)$ & $\mathrm{C} 18-\mathrm{C} 19-\mathrm{C} 20-\mathrm{C} 15$ & $-0.7(5)$ \\
\hline $\mathrm{C} 8-\mathrm{N} 1-\mathrm{C} 9-\mathrm{C} 10$ & $147.8(3)$ & $\mathrm{C} 16-\mathrm{C} 15-\mathrm{C} 20-\mathrm{O} 2$ & $179.1(3)$ \\
\hline $\mathrm{N} 1-\mathrm{C} 9-\mathrm{C} 10-\mathrm{C} 12$ & $-178.1(3)$ & $\mathrm{C} 14-\mathrm{C} 15-\mathrm{C} 20-\mathrm{O} 2$ & $-1.3(5)$ \\
\hline $\mathrm{N} 1-\mathrm{C} 9-\mathrm{C} 10-\mathrm{C} 13$ & $-57.2(4)$ & $\mathrm{C} 16-\mathrm{C} 15-\mathrm{C} 20-\mathrm{C} 19$ & $0.0(4)$ \\
\hline
\end{tabular}


supporting information

$\mathrm{N} 1-\mathrm{C} 9-\mathrm{C} 10-\mathrm{C} 11$

$60.9(4)$

$\mathrm{C} 14-\mathrm{C} 15-\mathrm{C} 20-\mathrm{C} 19$

$179.5(3)$

Hydrogen-bond geometry $\left(\AA,{ }^{\circ}\right)$

\begin{tabular}{lllll}
\hline$D-\mathrm{H} \cdots A$ & $D-\mathrm{H}$ & $\mathrm{H} \cdots A$ & $D \cdots A$ & $D-\mathrm{H} \cdots A$ \\
\hline $\mathrm{O} 1-\mathrm{H} 1 A \cdots \mathrm{N} 1$ & 0.82 & 1.89 & $2.620(3)$ & 147 \\
$\mathrm{O} 2-\mathrm{H} 2 A \cdots \mathrm{N} 2$ & 0.82 & 1.88 & $2.609(4)$ & 147 \\
\hline
\end{tabular}

